\title{
Positive emotion-specific changes in the gene expression profile of tickled rats
}

\author{
MIYO HORI $^{1 *}$, TAKASHI HAYASHI ${ }^{1 *}$, YOSHIMI NAKAGAWA $^{2}$, \\ SHIGEKO SAKAMOTO ${ }^{1}$, OSAMU URAYAMA ${ }^{2}$ and KAZUO MURAKAMI ${ }^{1}$
${ }^{1}$ Bio-Laboratory, Foundation for Advancement of International Science, 586-9 Akatsuka-Ushigafuchi, Tsukuba, Ibaraki 305-0062; ${ }^{2}$ Graduate School of Comprehensive Human Sciences, University of Tsukuba, 1-1-1 Tennoudai, Tsukuba, Ibaraki 305-8577, Japan

Received September 30, 2008; Accepted November 28, 2008

DOI: 10.3892/mmr_00000077

\begin{abstract}
The aim of this study was to investigate changes in gene expression after tactile stimulation (tickling) accompanied by positive emotion in the adolescent rat brain. We observed a positive emotional response $(50-\mathrm{kHz}$ ultrasonic vocalizations) after tickling using a modified version of the Panksepp method, and then comprehensively compared gene expression levels in the hypothalamus of the tickled rats and control rats using the microarray technique. After 4 weeks of stimulation, the expression levels of 321 of the 41,012 genes (including transcripts) were changed; 136 genes were up-regulated (>1.5-fold) and 185 were down-regulated $(<0.67$-fold) in the tickled rat group. Upon ontology analysis, the up-regulated genes were assigned to the following Gene Ontology (GO) terms: feeding behavior, neuropeptide signaling pathway, biogenic amine biosynthesis and catecholamine biosynthesis. Down-regulated genes were not assigned to any GO term categorized as a biological process. In conclusion, repeated tickling stimulation with positive emotion affected neuronal circuitry directly and/or indirectly, and altered the expression of genes related to the regulation of feeding in the adolescent rat hypothalamus.
\end{abstract}

\section{Introduction}

The mind and genes are considered to interact with each other $(1,2)$. We have demonstrated that laughter, an expression of positive emotion, influences the mind and body at a molecularbiological level (3-6). Studies concerning the physiological

Correspondence to: Dr Takashi Hayashi, Bio-Laboratory, Foundation for Advancement of International Science, 586-9 Akatsuka-Ushigafuchi, Tsukuba, Ibaraki 305-0062, Japan

E-mail: hayashi@fais.or.jp

${ }^{*}$ Contributed equally

Key words: gene expression, positive emotion, rat, brain, stimulation, vocalization effect of laughter were triggered by Cousins (7), who reported on his own recovery from disease through laughter. Since then, most studies have focused on the immunological aspects of laughter (8-10). We recently found that laughter suppressed an increase in the postprandial blood glucose level in patients with type 2 diabetes (3), and caused specific changes in gene expression in peripheral blood leucocytes (4). Laughter upregulated the expression of genes ameliorating the progression of diabetic microvascular complications (5) and improving glucose intolerance by modulating NK cell activity (6). Positive emotional expressions such as laughter are the result of neurotransmission, but how this process is related to glucose metabolism has not been clarified. Therefore, we focused on changes in gene expression initiated by positive emotion in the hypothalamus as the regulatory center of blood glucose level.

Rats exhibit specific ultrasonic vocalizations (USVs) in response to various social interactions and stimulation, with $50-\mathrm{kHz}$ USVs reflecting positive emotional states, proposed as an evolutionary antecedent to human joy (11). This $50-\mathrm{kHz}$ USV index of positive emotion was identified by Panksepp, who also established a positive stimulus administration method that mimicks the rough-and-tumble play of rats $(12,13)$. In the present study, we analyzed changes in gene expression after tactile stimulation (tickling) accompanied by positive emotion using the microarray technique. This is the first study to demonstrate that repeated tactile stimulation affects neuronal circuitry and alters the expression of many genes in the rat hypothalamus.

\section{Materials and methods}

Subjects. Twenty-one day old post-weaning male Wistar rats (Japan SLC Inc., Shizuoka, Japan) were used. To avoid ludic behavior, such as rough-and-tumble play, animals were individually housed in standard polycarbonate cages (W270 x L440 x H187 mm) with wood chip bedding. Rats were allowed ad libitum access to water and food and maintained under specific pathogen-free conditions at a room temperature of $21 \pm 1^{\circ} \mathrm{C}, 50-60 \%$ humidity, and a $12: 12 \mathrm{light} /$ dark cycle (lights on at 7:00) during the study period. After 1 week of acclimatization, the rats were weighed and then divided into two groups: the tickled group and, as the control, the light- 
touched group ( $\mathrm{n}=4$ each). The body weights of rats in the tickled and light-touched groups were $85.6 \pm 4.8$ and $86.0 \pm 2.1 \mathrm{~g}$ (means $\pm \mathrm{SD})$, respectively. Animals received stimulation once a day for 5 consecutive days (Monday-Friday) for 4 weeks. The experimental procedures were approved by the Institutional Animal Experiment Committee of the University of Tsukuba.

Stimulation. A modified version of the Panksepp method (12) was used for tickling stimulation. Rats were individually transferred to a test box (W270 x L440 x H187 mm, with floor and four sides covered with black felt). After a 15 -sec stimulation-free period, tickling stimulation was administered for $15 \mathrm{sec}$. This procedure was repeated four times (one tickling session). This method mimics the dorsal contact and pinning behavior of the rough-and-tumble play of rats (14). The rat was grasped on the dorsal side and tickled on the posterior neck with fingers, rapidly overturned and vigorously tickled over the abdomen while being pushed onto the floor in a supine position, and then released. After the first tickling session, there was a 1-min rest period, followed by the second session (5 min in total).

Light-touch stimulation, which serves as a discernible stimulation (12), was adopted as the control in this study. Rats were gently touched on the dorsal region every $3 \mathrm{sec}$ instead of undergoing the 15 -sec tickling stimulation. The timing of stimulation was the same as that outlined above.

Ultrasonic vocalization recording and fast Fourier transform analysis. To analyze the index of positive emotion in rats, $50-\mathrm{kHz}$ USVs were recorded for each rat during stimulation and resting using a high-frequency microphone (MI-3140, Ono Sokki Co., Kanagawa, Japan) suspended $25 \mathrm{~cm}$ above the floor of the test box. Frequency component analysis (hanning window; frequency range, 10-100 kHz; sampling frame length, 4096 points; average, power spectrum peak hold) was performed using an ultra high-band acoustic analysis system (DS-2100, Ono Sokki Co.). After Fourier transformation of vocal sound, the peak sound pressure levels (dB) of the frequency components in every 15 -sec period were plotted on a graph for analysis.

Approach latency. To analyze the index of positive behavior reinforcement, the rats were tested for approach latency to tactile stimulation. After two tickling (or light-touch) sessions, rats were placed in a corner of the test box and the period until the rat approached and touched the experimenter's hand to receive tickling (or light-touch) was recorded. The maximum 30-sec latency was recorded.

Sample collection and preparation. On the day following the final stimulation session at the end of the 4-week period, rats aged 8 weeks were perfused with saline and decapitated under ether anesthesia, and their brains were excised by posterior craniotomy. Each excised brain was rapidly dissected according to the method of Glowinski and Iversen (15), and the hypothalamus was obtained. Hypothalamic tissues were suspended in RNAlater RNA Stabilization Reagent (Qiagen $\mathrm{GmbH}$, Hilden, Germany) and stored at $-80^{\circ} \mathrm{C}$. Total RNA was prepared from stored tissue specimens using an RNeasy Mini Kit (Qiagen $\mathrm{GmbH}$ ) following the manufacturer's protocol.
Microarray hybridization and data acquisition. Equal amounts of total RNA preparations from the four rats were mixed in each group and comprehensively analyzed by the microarray technique. cDNA was synthesized using 500 ng of total RNA and T7 RNA promoter sequence-bound Oligo (dT)24 as the primer. Subsequently, aminoallyl nucleotide-incorporated cRNA was prepared by in vitro transcription. This cRNA was labeled with a fluorescent dye, cyanine $(\mathrm{Cy}) 3$ or Cy5 $(16,17)$. Equal amounts of Cy3-labeled cRNA derived from the lighttouched group and Cy5-labeled cRNA derived from the tickled group were mixed. The mixture was applied to a Whole Rat Genome Oligo Microarray (G4131A, Agilent Technologies, CA, USA) on which 41,012 genes (including transcripts) were spotted, and hybridization was allowed to proceed for $17 \mathrm{~h}$ at $65^{\circ} \mathrm{C}$ as described by the manufacturer. After hybridization, the arrays were washed and scanned using a confocal laser scanner (Agilent G2565BA). The fluorescence intensities on the scanned images were quantified. Background correction and normalization were performed as previously reported (8). Data were registered in the Gene Expression Omnibus (National Center for Biotechnology Information) with accession no. GSE11267. Genes with 1.5- and 0.67-fold differences in expression level in the tickled group compared to the lighttouched group were discriminated.

Gene ontology analysis. The discriminated genes were examined for their biological meaning based on gene ontology (18). Gene ID and GO were collated using the Biological Network Gene Ontology tool, and GO hierarchies were drawn using Cytoscape (19). Each GO term was analyzed to determine whether the frequency in the discriminated gene group was elevated compared to that in all genes on the microarray using the hypergeometric test at a false discovery rate (FDR) of $<0.1$. GO terms judged significant by this examination were regarded as specific terms for discriminated genes.

\section{Results}

Behavioral analysis. All rats in the tickled group emitted a sign of positive emotion at a frequency of $\sim 50 \mathrm{kHz}$ during the tickling sessions from the initial session on the first day, and did not emit $\sim 20 \mathrm{kHz}$ USVs, an aversive sign of stress or negative emotion, at any time during the experimental period (Fig. 1A and B). This is consistent with the previous study (12). In contrast, no such $50-\mathrm{kHz}$ USVs were noted in the lighttouched group throughout the experimental period, though the light-touched rats emitted $20-\mathrm{kHz}$ USVs on only the first day (Fig. 1C and D). The approach latencies on the first day of the tickled and the light-touched groups were 5.5 $\pm 5.75 \mathrm{sec}$ $($ mean $\pm \mathrm{SD})$ and $>30 \mathrm{sec}$, respectively. These data are consistent with the previous study, demonstrating that tickling positively reinforces tactile stimulation (12).

Gene expression analysis. Repeated stimulation with tickling altered the expression of many genes in the hypothalamus of the adolescent rats. After the 4-week stimulation, the expression levels of 321 of the 41,012 genes (including transcripts) were altered in the tickled group compared to the lighttouched groups; 136 genes were up-regulated ( $>1.5$-fold) and 185 were down-regulated $(<0.67$-fold $)$. 
A

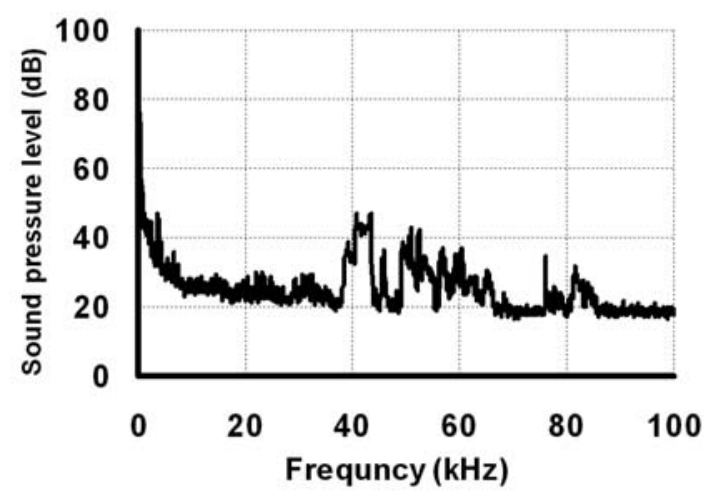

B

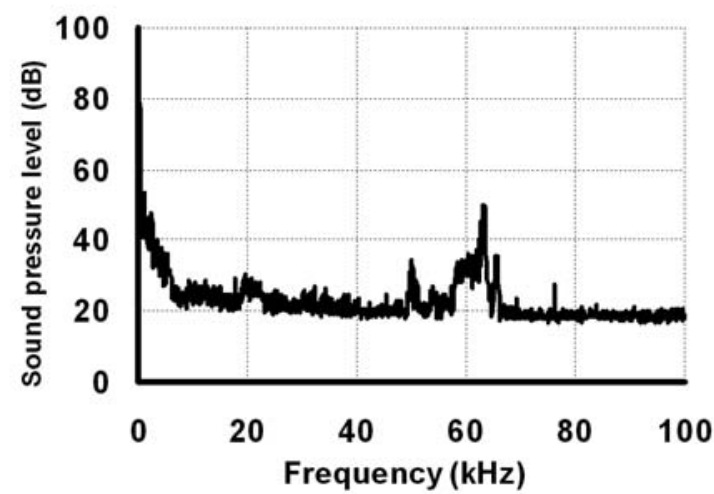

C

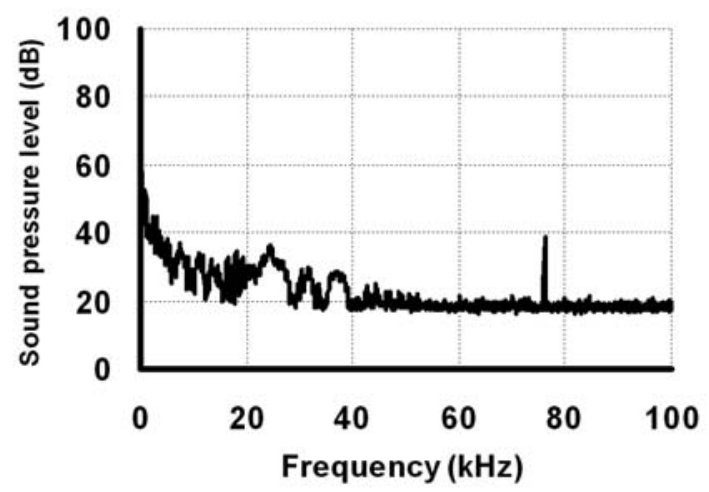

D

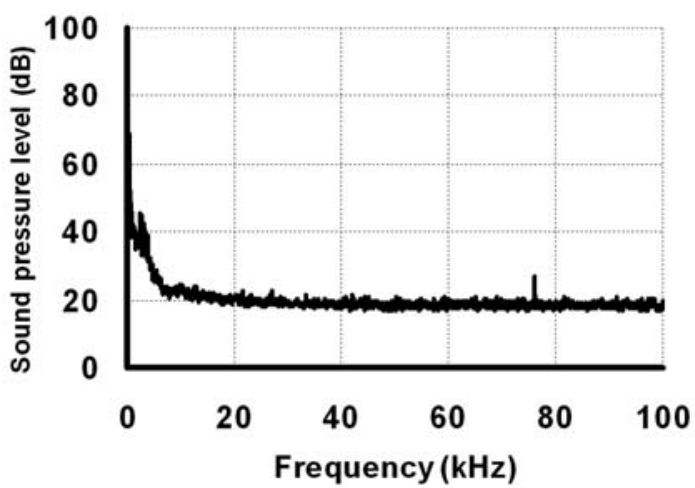

Figure 1. Power spectra of the ultrasonic vocalizations emitted by rats. After FFT analysis, data were plotted as the peak of sound pressure levels during $15 \mathrm{sec}$ of stimulation in the tickled group on the first day (A) and after 4 weeks (B), and in the light-touched group on the first day (C) and after 4 weeks (D).

The up-regulated genes were assigned $46 \mathrm{GO}$ terms categorized as biological processes (Fig. 2). The GO terms with significant enrichment (FDR $<0.01)$ were feeding behavior (GO ID 7631), neuropeptide signaling pathway (GO ID 7218), biogenic amine biosynthesis (GO ID 42401) and catecholamine biosynthesis (GO ID 42423). The genes encoding galanin-like peptide precursor (Galp 2.14-fold), pro-opiomelanocortin (Pomc, 2.13-fold), pro-melaninconcentrating hormone (Pmch, 1.87-fold), agouti-related protein homolog (Agrp, 1.80-fold), orexin (hypocretin; Hcrt, 1.60-fold) and neuropeptide Y (Npy, 1.59-fold) were assigned feeding behavior terms, and Pomc, Agrp, Npy, Galp, Pmch and cocaine and amphetamine-regulated transcript (Cart, 1.94-fold) were assigned neuropeptide signaling pathway terms. The genes encoding histidine decarboxylase (Hdc, 1.80 -fold) and tyrosine hydroxylase (Th, 1.58-fold) were assigned catecholamine biosynthesis terms. Down-regulated genes were not assigned to any GO term categorized as a biological process.

\section{Discussion}

The objective of this study was to investigate changes in gene expression precipitated by tactile stimulation accompanied by positive emotion in the brain. Repeated stimulation altered the expression of many genes in the hypothalamus of adoles- cent rats. Ontology analysis of the genes that underwent a marked increase in expression revealed a significant enrichment in those assigned feeding behavior terms within the GO category of biological processes, namely the genes Agrp, Hcrt, Npy, Galp, Pmch and Pomc. All these genes, with the exception of Hcrt, were also assigned neuropeptide signaling pathway terms. Positive emotion induced the expression of genes encoding feeding behavior-related neuropeptides. The most important brain region related to feeding behavior is the hypothalamus. Within the hypothalmus, the feeding center is located in the lateral hypothalamic area (LHA) and the satiety center is in the ventromedial hypothalamic nucleus (VMH) $(20,21)$. AgRP, HCRT, NPY, GALP, PMCH and POMC are substances which regulate feeding; the first five promote feeding behavior (22-25), while POMC is precursor for $\alpha$ melanocyte-stimulating hormone ( $\alpha \mathrm{MSH})$, which suppresses it (26). These substances interact with each other and control feeding and energy expenditure $(23,26)$, suggesting that positive stimulation followed by the process of neurotransmission regulates the function of these genes in the central nervous system. HCRT is also involved in arousal, sleep and emotion (27).

Hdc encodes histidine decarboxylase (HDC), which is involved in histamine synthesis, and Th encodes tyrosine hydroxylase $(\mathrm{TH})$, which is the rate-limiting enzyme of catecholamine synthesis, such as dopamine synthesis. In the 


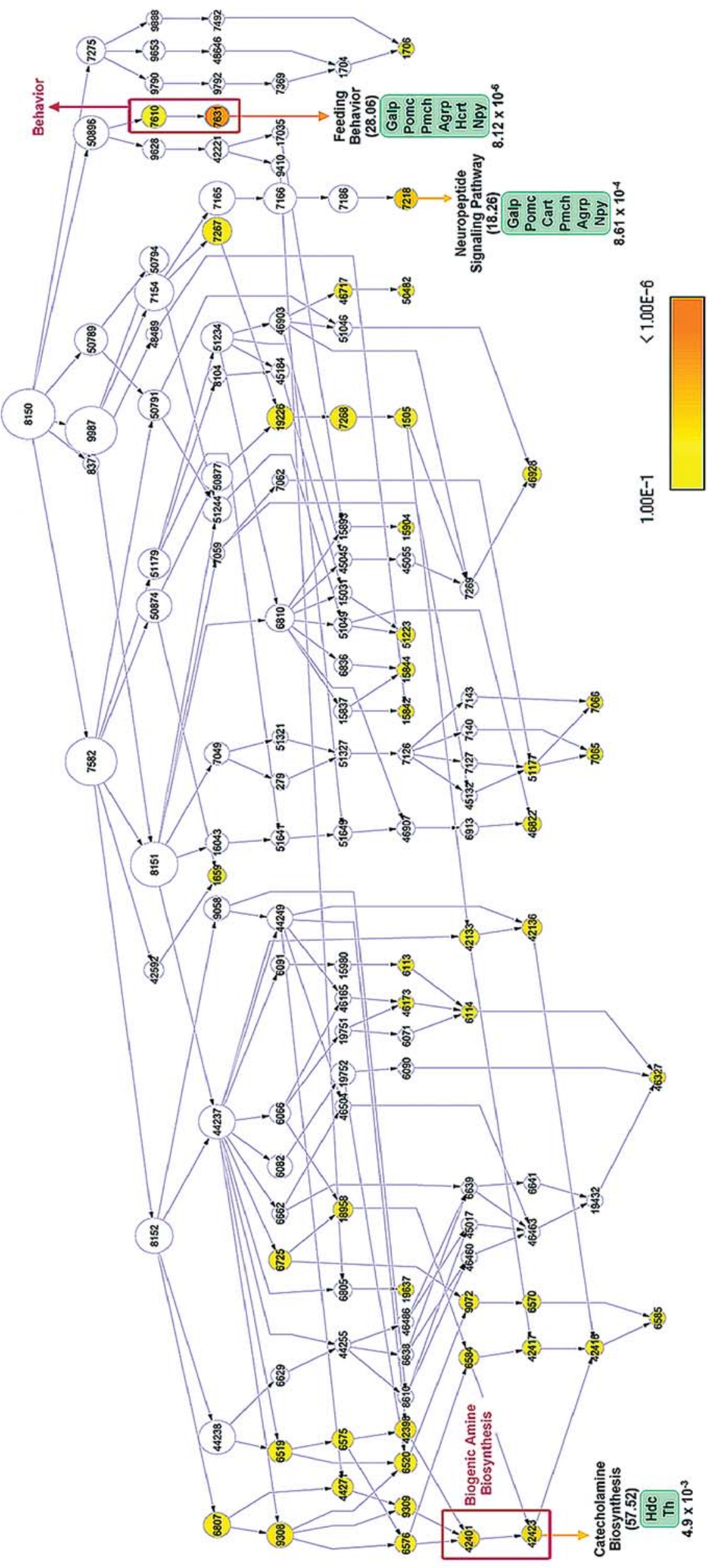

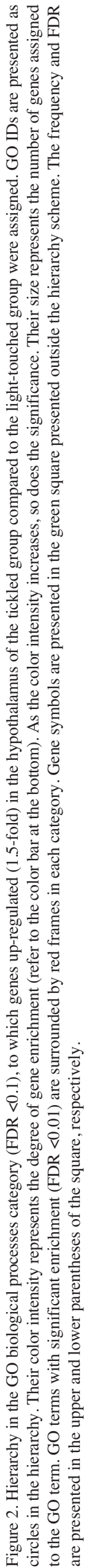


various functions of monoamines, the association of dopamine and histamine with feeding behavior, such as the promotion and suppression of feeding, respectively, has also been reported $(28,29)$.

In conclusion, repeated tickling stimulation with positive emotion affected neuronal circuitry directly and/or indirectly, and altered expression of the genes related to feeding regulation in the adolescent rat hypothalamus. It is known that the food intake system in the LHA is driven by a stomach-empty signal (26). Further analysis is necessary to investigate how tickling modulates local expression of feeding-regulation genes and, as a result, the blood glucose level.

\section{Acknowledgements}

We are grateful to H. Shudoh for excellent technical support. We especially thank Dr Y. Ichitani for his helpful technical guidance and critical advice on experimental design. This study was supported by a grant from the Society for Mind-Heart and Gene at the Foundation for the Advancement of International Science. M. Hori and T. Hayashi were responsible for the study design, execution, data analysis and preparation of the manuscript.

\section{References}

1. Rossi EL: The Psychobiology of Mind-Body Healing: New Concepts of Therapeutic Hypnosis. WW Norton, New York, 1993.

2. Murakami K and Hayashi T: Interaction between mind-heart and gene. J Intl Soc Life Info Sci 20: 122-130, 2002.

3. Hayashi K, Hayashi T, Iwanaga S, Kawai K, Ishii H, Shoji S and Murakami K: Laughter lowered the increase in postprandial blood glucose. Diabetes Care 26: 1651-1652, 2003.

4. Hayashi T, Urayama O, Kawai K, Hayashi K, Iwanaga S, Ohta M, Saito T and Murakami K: Laughter regulates gene expression in patients with type 2 diabetes. Psychother Psychosom 75: 62-65, 2006.

5. Hayashi T, Urayama O, Hori M, Sakamoto S, Nasir UM, Iwanaga S, Hayashi K, Suzuki F, Kawai K and Murakami K Laughter modulates prorenin receptor gene expression in patients with type 2 diabetes. J Psychosom Res 62: 703-706, 2007.

6. Hayashi T, Tsujii S, Iburi T, Tamanaha T, Yamagami K, Ishibashi R, Hori M, Sakamoto S, Ishii H and Murakami K: Laughter up-regulates the genes related to NK cell activity in diabetes. Biomed Res 28: 281-285, 2007.

7. Cousins N: Anatomy of an illness (as perceived by the patient). N Engl J Med 295: 1458-1463, 1976.

8. Berk LS, Felten DL, Tan SA, Bittman BB and Westengard J: Modulation of neuroimmune parameters during the eustress of humor-associated mirthful laughter. Altern Ther Health Med 7: 62-72, 74-76, 2001.

9. Nakajima A, Hirai $\mathrm{H}$ and Yoshino $\mathrm{S}$ : Reassessment of mirthful laughter in rheumatoid arthritis. J Rheumatol 26: 512-513, 1999.

10. Takahashi K, Iwase M, Yamashita K, Tatsumoto Y, Ue H Kuratsune H, Shimizu A and Takeda M: The elevation of natural killer cell activity induced by laughter in a crossover designed study. Int J Mol Med 8: 645-650, 2001.
11. Panksepp J and Burgdorf J: "Laughing" rats and the evolutionary antecedents of human joy? Physiol Behav 79: 533-547, 2003.

12. Burgdorf J and Panksepp J: Tickling induces reward in adolescent rats. Physiol Behav 72: 167-173, 2001.

13. Burgdorf J, Panksepp J, Brudzynski SM, Kroes R and Moskal JR: Breeding for $50-\mathrm{kHz}$ positive affective vocalization in rats. Behav Genet 35: 67-72, 2005.

14. Knutson B, Burgdorf J and Panksepp J: Ultrasonic vocalizations as indices of affective states in rats. Psychol Bull 128: 961-977, 2002.

15. Glowinski J and Iversen LL: Regional studies of catecholamines in the rat brain. I. The disposition of $\left[{ }^{3} \mathrm{H}\right]$ norepinephrine, $\left[{ }^{3} \mathrm{H}\right]$ dopamine and $\left[{ }^{3} \mathrm{H}\right]$ dopa in various regions of the brain. J Neurochem 13: 655-669, 1966.

16. Luo L, Salunga RC, Guo H, Bittner A, Joy KC, Galindo JE, Xiao H, Rogers KE, Wan JS, Jackson MR and Erlander MG: Gene expression profiles of laser-captured adjacent neuronal subtypes. Nat Med 5: 117-122, 1999.

17. Hughes TR, Mao M, Jone AR, Burchard J, Marton MJ, Shannon KW, Lefkowitz SM, Ziman M, Schelter JM, Meyer MR, Kobayashi S, Davis C, Dai H, He YD, Stephaniants SB, Cavet G, Walker WL, West A, Coffey E, Shoemaker DD, Stoughton R, Blanchard AP, Friend SH and Linsley PS: Expression profiling using microarrays fabricated by an ink-jet oligonucleotide synthesizer. Nat Biotechnol 19: 342-347, 2001.

18. Ashburner M, Bal CA, Blake JA, Botstein D, Butler H, Cherry JM, Davis AP, Dolinski K, Dwight SS Eppig JT, Harris MA, Hill DP, Issel-Tarver L, Kasarskis A, Lewis S, Matese JC, Richardson JE, Ringwald M, Rubin GM and Sherlock G: Gene ontology: tool for the unification of biology. The Gene Ontology Consortium. Nat Genet 25: 25-29, 2000.

19. Maere S, Heymans K and Kuiper M: BiNGO: a Cytoscape plugin to assess overrepresentation of gene ontology categories in biological networks. Bioinformatics 21: 3448-3449, 2005.

20. Anand BK and Brobeck JR: Localization of a "feeding center" in the hypothalamus of the rat. Proc Soc Exp Biol Med 77: 323-324, 1951.

21. Stellar E: The physiology of motivation. Psychol Rev 101: 301-311, 1994.

22. Kuramochi M, Onaka T, Kohno D, Kato S and Yada T: Galaninlike peptide stimulates food intake via activation of neuropeptide $\mathrm{Y}$ neurons in the hypothalamic dorsomedial nucleus of the rat. Endocrinology 147: 1744-1752, 2006.

23. Schwartz MW, Woods SC, Porte D Jr, Seeley RJ and Baskin DG: Central nervous system control of food intake. Nature 404: 661-671, 2000.

24. Shi Y: Beyond skin color: emerging roles of melanin-concentrating hormone in energy homeostasis and other physiological functions. Peptides 25: 1605-1611, 2004.

25. Yamanaka A, Kunii K, Nambu T, Tsujino N, Sakai A, Matsuzaki I, Miwa Y, Goto K and Sakurai T: Orexin-induced food intake involves neuropeptide Y pathway. Brain Res 859: 404-409, 2000.

26. Konturek PC, Konturek JW, Czesnikiewicz-Guzik M, Brzozowski T, Sito E and Konturek SJ: Neuro-hormonal control of food intake: basic mechanisms and clinical implications. J Physiol Pharmacol 56 (Suppl 6): 5-25, 2005.

27. Chemelli RM, Willie JT, Sinton CM, Elmquist JK, Scammell T, Lee C, Richardson JA, Williams SC, Xiong Y, Kisanuki Y, Fitch TE, Nakazato M, Hammer RE, Saper CB and Yanagisawa M: Narcolepsy in orexin knockout mice: molecular genetics of sleep regulation. Cell 98: 437-451, 1999.

28. Meguid MM, Fetissov SO, Varma M, Sato T, Zhang L, Laviano A and Rossi-Fanelli F: Hypothalamic dopamine and serotonin in the regulation of food intake. Nutrition 16: 843-857, 2000.

29. Morimoto T, Yamamoto $Y$ and Yamatodani A: Brain histamine and feeding behavior. Behav Brain Res 124: 145-150, 2001. 